\title{
Study on the Separation Technology of Temperature and Emissivity Data Based on the Airborne Thermal Infrared Image
}

\author{
Junhu Wang
}

\begin{abstract}
Since the high-resolution airborne thermal infrared imaging spectrometer is used to civilian field, it shows attractive application prospect in the field of objects recognition. Taking an area for example, the paper carries out the technology research for temperature and emissivity separation, based on the highresolution aerial thermal infrared image obtained in the early morning, and completes the subjective visual evaluation and objective statistical evaluation for the temperature and emissivity image separated by different methods from aerial thermal infrared image. Furthermore, the paper establishes a set of temperature and emissivity separation and evaluation methods which are suitable for anomaly heat identification. Above all, the temperature and emissivity image separated by proper methods will provide accurate data support for objects identification.
\end{abstract}

Keywords: Thermal infrared image - Temperature and emissivity separation . Objects identification . Support

\subsection{Introduction}

The application research on the thermal infrared remote sensing are not well developed, although imaging spectral technique is a powerful tool in collecting and analyzing environmental data. With the development of remote sensing technology, more thermal infrared sensors were developed, and more attention was paid to thermal infrared data [1]. The emissivity and temperature retrieved from thermal infrared data will play an extremely important role for scientific research and operational application. Especially for the field of earth science, the technology of thermal infrared remote sensing has shown that there is great potential in the objects recognition, because it could detect the weak thermal anomaly . The

\footnotetext{
$1_{\text {Junhu Wang }(\bowtie)}$

Beijing Research Institute of Uranium Geology, National Key Laboratory of Remote Sensing Information and Image Analysis Technology ,Beijing China

e-mail: tcwjh2001@163.com
} 
emissivity information is an important parameter, and each object has unique emissivity characteristics, and hence, emissivity spectra can be used to distinguish different objects. Land surface temperature is an important parameter for understanding land surface processes, Any object emits infrared radiation all the time as long as its temperature is above absolute zero. Objects hid in the background always has the different temperature from background and can be identified by thermal imaging equipment. Therefore, temperature and emissivity separation is key variable for infrared remote sensing applied to identify objects.

At present, thermal infrared data acquired by ASTER sensor is widely used in the application fields mostly, and the method of temperature and emissivity separation (TES) is provided by ASTER official website, It is proved that the emissivity error is below 0.1 using ASTER simulated data [2]. Schmugge applied TES method to retrieve temperature information of grassland based on the thermal infrared multispectral data, and proved that the temperature error is about 3K[3]. Although scholars carried out wide application research based on ASTER thermal infrared data, the spatial resolution of ASTER thermal infrared data is 90 meters, and there are five bands in the thermal infrared bands. The low spatial and spectral resolution greatly limited the application effect. With the development of hardware technology of sensor, hyper-spectral thermal infrared data has created favorable conditions for research on the emissivity and temperature of objects, because of hyper-spectral and high spatial resolution, and the fine emissivity features of objects improve the retrieval accuracy of temperature. Thermal Airborne Hyperspectral Imager (TASI) is one of the most advanced airborne thermal infrared imagers created by Canada, there are 32 bands in the thermal infrared region $(8 \mu \mathrm{m}$ $-11.5 \mu \mathrm{m}$ ), the wavelength spacing is $0.1095 \mu \mathrm{m}$, the FWHM (Full width at half maximum) is $0.0548 \mu \mathrm{m}$, and total fields of view is $40^{\circ}$ [4].The new thermal infrared image with high resolution acquired by TASI provides the possibility to separate temperature and emissivity spectral with good precision.

Based on the background mentioned above, taking an area for example, the paper studied the separation methods of temperature and emissivity spectrum which could achieve the good precision, based on high resolution thermal infrared image of TASI. The paper expected that all of work could provide the temperature and emissivity image with high quality to identify objects accurately.

\subsection{Thermal Infrared Data Preprocessing}

It is necessary to do the radiance calibration for every band of TASI data before collecting data, in order to acquire the objective radiance. In other words, the digital number value (DN) recorded by the thermal infrared imaging spectrometer should be transferred to spectral radiance value. The radiance calibration method of TASI data is implemented by the means of measuring two standard blackbody with known temperature and emissivity, based on the assumption of a linear sen- 
sor response function. First of all, it is assumed that there is the linear relationship between the digital number value recorded by TASI and the radiance, and then radiance calibration band by band can be calculated as follows:

$$
\mathrm{L}=\mathrm{C}_{1} \times \mathrm{DN}+\mathrm{C}_{2}
$$

Where $c_{1}$ and $c_{2}$ is gain and offset of instrument spectral response function, which could be known from TASI instruction. The progress of radiance calibration can be finished by the IDL programming.

Atmospheric effects in general can't be ignored in the radiation temperature and features emissivity measurement. It makes thermal infrared signal distort which is recorded by sensor. As a result, remote sensing information deviates from the ground truth. Therefore, the signal received by thermal infrared sensor consists of two parts. One part of radiation is its thermal radiation emitted by measured objects, another part is emitted from surrounding environment. Two parts are mixed as the apparent temperature [5]. To retrieve the temperature and emissivity accurately, apparent radiance temperature is not enough, further atmospheric correction must be made to remove the atmospheric effects.

The paper adopted the In-Scene Atmospheric Compensation Algorithms (ISAC), because atmospheric parameters synchronizing with thermal infrared data of TASI are not easily available, and the emissivity data only requires higher relative value rather than absolute value. This algorithm assumes that the atmosphere above data source is homogeneous, and surface objects are blackbody without downlink radiation. Atmospheric uplink radiation and atmospheric transmission could be approximately calculated in accordance with the following method: First of all, the surface temperature of each pixel is estimated from data, then the brightness temperature is estimated by the Planck formula assuming emissivity is equal to one. Furthermore, the scatter diagram of radiation rate and the brightness temperature are drawn using one of two methods above in order to determine a curve. As a result, atmospheric uplink radiation could be calculated from the tilt and offset of curve [6]. Based on the above principle, the paper completed the atmospheric correction by IDL programming language.

\subsection{The Algorithm Search of Temperature and Emissivity Data Separation}

Thermal infrared radiation acquired by sensor is the coupling function of surface temperature and emissivity. Therefore, it needs to separate temperature and emissivity information from the thermal infrared radiation. The core issue of separation is that if emissivity is unknown completely, the number of data is $\mathrm{N}$ in the $\mathrm{N}$ bands observation, and the number unknown is N. However, the object temperature is unknown too, so the separation method must solve $\mathrm{N}+1$ parameters with $\mathrm{N}$ equations. In order to solve the underdetermined equation, additional condition must be introduced. Additional conditions are usually that the researchers have 
certain prior knowledge on the emissivity spectral of objects. The different prior knowledge decides different separation algorithm. At present, the separation algorithms conclude Reference Channel Method (RCM), Emissivity Normalization Method(NEM), Alpha Residuals Method(ARM) and so on[7].

Reference Channel Method (RCM) is first proposed by Kahle [8]. This approach assumes that the emissivity in the certain band of objects can be obtained by prior knowledge. Therefore, the real temperature of objects can be calculated through the radiance value of this band, and the emissivity of other bands can be calculated correspondingly. Considering the environmental radiation, the formula of REM is as follows:

$$
\begin{aligned}
& T=B^{-1}\left(\frac{L_{r}-\left(1-\varepsilon_{r} L_{a t m \downarrow}(r)\right)}{\varepsilon_{r}}\right)=c_{2} /\left[\lambda \lg \left(\frac{c_{1} \varepsilon_{r}}{\lambda^{5} \pi\left(L_{r}-\left(1-\varepsilon_{r}\right) L_{a t m \downarrow}(r)\right.}+1\right)\right] \\
& \varepsilon(\lambda)=\left[L(\lambda, T)-L_{a t m \downarrow}(\lambda, T)\right] /\left[B(\lambda, T)-L_{a t m \downarrow}(\lambda, T)\right]
\end{aligned}
$$

$\mathrm{T}$ represents the blackbody temperature calculated by different wavelength $(\lambda)$ in case that $\varepsilon_{\mathrm{r}}$ is known. $L(\lambda)$ represents the radiance value of different bands acquired by sensor, $B(\lambda, T)$ stands for the radiance value of blackbody, $L_{\text {atm }}$ stands for the atmospheric downward radiance, $\mathrm{c}_{1}=3.7418 \times 10^{-16} \mathrm{~W} \cdot \mathrm{m}^{2}$, $\mathrm{c}_{2}=14388 \mu \mathrm{m} \cdot \mathrm{K}$.

The paper completed the separation of temperature and emissivity, based on the TASI image in an area by this algorithm. The second band (wavelength range is among 8.11-8.22 $\mu \mathrm{m}$ ) of TASI data was chosen as the reference band, the reference emissivity is 8.164 , according to the emissivity experience value of objects. The result is shown in Figure1.1(B) and Figure1(D).

Alpha Residuals Method (ARM) is first proposed by Keakt and Gabell. The algorithm uses the Wien's approximation to Planck's law, ignores the minus 1 in the denominator of formula 2, As a result, it gets an approximate linear expression by taking the logarithm for Planck formula[9]. For the blackbody whose temperature is $300 \mathrm{~K}$, it causes about $1 \%$ error at the wavelength of $10 \mu \mathrm{m}$ using Venn approximation. The specific formula is as follows:

$\lambda_{j} \ln \varepsilon_{j}-\frac{1}{N} \sum_{j=1}^{N} \lambda_{j} \ln \varepsilon_{j}=\lambda_{j} \ln \varepsilon_{j}-\frac{1}{N} \sum_{j=1}^{N}\left(\lambda_{j} \ln L_{j}-\lambda_{j} \ln c_{1}\right)+\frac{1}{N} \sum_{j=1}^{N}\left(\lambda_{j} \ln c_{1}-5 \lambda_{j} \ln \lambda_{j}\right)-\frac{5}{N} \sum_{j=1}^{N}\left(\lambda_{j} \ln \lambda_{j}+\lambda_{j} \ln \pi\right)-\frac{\ln \pi}{N} \sum_{j=1}^{N} \lambda_{j}$

This equation does not contain the temperature $(\mathrm{T})$, therefore, a new parameter $\mathrm{j}$ is defined, it is called the $\alpha$ remaining (formula 5). It is not relevant to the temperature $(\mathrm{T})$.

$$
\alpha_{j}=\lambda_{j} \ln \varepsilon_{j}-\frac{1}{N} \sum_{j=1}^{N} \lambda_{j} \ln \varepsilon_{j}=\lambda_{j} \ln \lambda_{j}-\frac{1}{N} \sum_{j=1}^{N}\left(\lambda_{j} \ln L_{j}+K_{j}\right)
$$

In the formula, $\mathrm{K}_{\mathrm{j}}$ is not relevant to the radiance, and can be calculated as a constant. Thus, $\alpha_{j}$ of every band may be calculated by radiance $\mathrm{L}_{\mathrm{j}}$. The $\alpha_{j}$ remaining actually reflects parameters of emissivity spectral curve. The shape of emissivity curve will be known by measuring the radiance spectral curve of objects. The method is widely used, especially for the 
rock and mineral identification. However, the emissivity spectral shape of objects could only be known, but not its absolute value. The average needs to be known in order to determine the exact value of emissivity, this average can only be known by other experience constraints. The paper separated the emissivity from TASI image of an area by this algorithm, the result is shown in Figure1.1(E).

The principle of Normalized Emissivity Method (NEM) is to calculate the temperature of each pixel by constant emissivity, the maximum temperature of each pixel is used in the Planck formula to calculate the emissivity. The specific idea is to assume that there is a band whose emissivity could reach the maximum ( $\varepsilon_{\max }$ ) for the high-spectral observation, and hence gray body's radiance of maximum ( $\varepsilon_{\max }$ ) can be used for the envelope. Special steps are as follows:

At first, the blackbody temperature corresponding to the radiance measured by all bands is calculated, the formula without considering the environment radiance is as follows :

$$
T_{\lambda}=B^{-1}\left(\frac{L(\lambda)}{\varepsilon_{\max }}\right)=c_{2} /\left[\lambda \lg \left(\frac{c_{1} \varepsilon_{\max }}{\lambda 5 \pi L(\lambda)}\right)+1\right]
$$

Where $T_{\lambda}$ is the blackbody temperature calculated by different wavelength, in case that $\varepsilon_{\text {max }}$ is constant. $L(\lambda)$ is the radiance obtained by different bands of sensor, $B(\lambda, T)$ is the radiance of blackbody, $\mathrm{c}_{1}=3.7418 \times 10^{-16} \mathrm{~W} \cdot \mathrm{m}^{2}, \mathrm{c}_{2}=14388 \mu \mathrm{m} . \mathrm{K}$. Secondly, the highest blackbody temperature of all bands is determined.

$$
T_{\lambda}=\max \left(T_{\lambda}\right)
$$

At last, the emissivity of all bands is calculated in the case that temperature mentioned above is true temperature of objects.

$$
\varepsilon(\lambda)=L(\lambda) / B(\lambda, T)
$$

Considering the environmental radiation $\left(L_{a t m \downarrow}\right)$, the formula is as follows:

$$
\begin{gathered}
T_{\lambda}=B^{-1}\left(\frac{L(\lambda)-\left(1-\varepsilon_{\max } L_{a t m \downarrow}(\lambda, T)\right)}{\varepsilon_{\max }}\right)=c_{2} /\left[\lambda \lg \left(\frac{c_{1} \varepsilon_{\max }}{\lambda^{5} \pi\left(L(\lambda)-\left(1-\varepsilon_{\max }\right) L_{a t m \downarrow}(\lambda, T)\right)}+1\right)\right] \\
T_{\lambda}=\max \left(T_{\lambda}\right) \\
\varepsilon(\lambda)=\left[L(\lambda)-L_{a t m \downarrow}(\lambda, T)\right] /\left[B(\lambda, T)-L_{a t m \downarrow}(\lambda, T)\right]
\end{gathered}
$$

The method of NEM is not complicated, and the principle is also more reasonable. There is greater practical value especially for combining with the experience. The paper used NEM method to separate the temperature and emissivity from TASI image of an area in the case that the maximum emissivity of objects is 0.98. The result is shown in Figure1.1(C) and Figure1(F). 


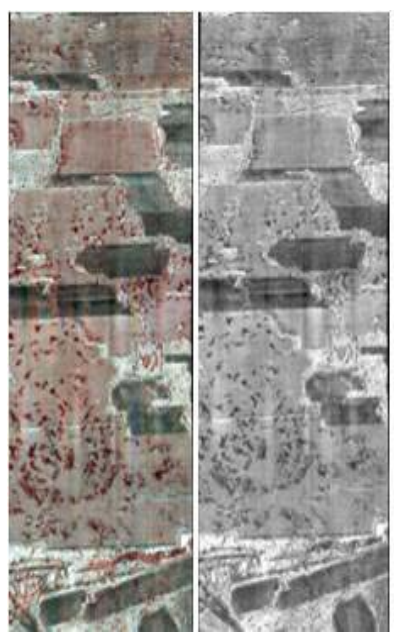

A

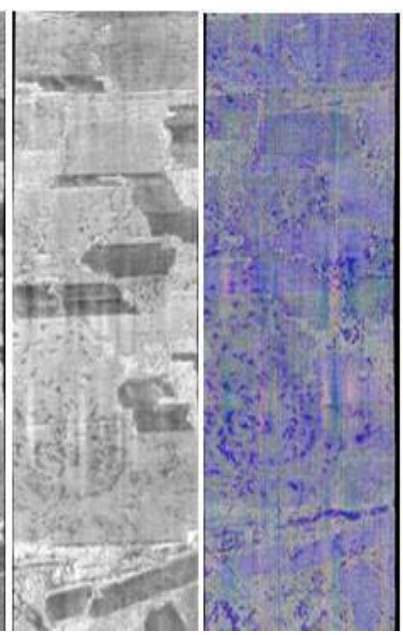

C

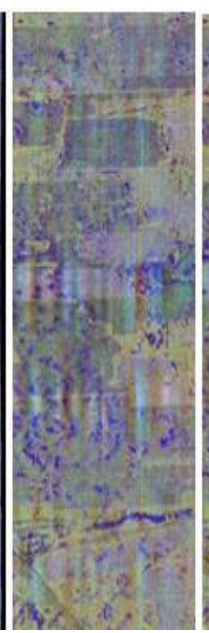

E

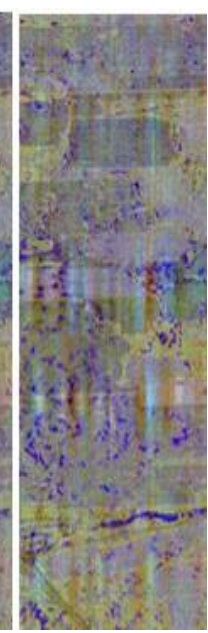

$\mathrm{F}$

Fig. 1.1 Color composite map of TASI 24(R)13(G)4(B) (A); temperature inversion map by RCE (B); temperature inversion map by NEM (C);emissivity inversion and color composite map of TASI emssivity14(R)7(G)5(B) by RCE (D); by $\alpha$ remaining method(E); by NEM (F)

\subsection{Results and Evaluation}

The paper adopted subjective visual evaluation, objective statistical evaluation to analyze the temperature and emissivity separated by three methods mentioned above. Details are as follows:

From Figure 1.1B and 1C, it can be seen that gray levels of temperature image separated by RCM are more plentiful than the temperature image separated by NEM, it is better to reflect changes in the brightness of the image caused by the weak temperature difference, and the strip noise is less. However, most of temperature value retrieved by RCM are between 1900K-2100K, the absolute error of temperature is larger. The temperature value retrieved by NEM are between 280K-294K, the temperature is consistent with the fact. Whereas, the temperature difference of different pixel is smaller, it is not conducive to objects identification with weak temperature difference. From Figure 1D, 1E and 1F, it can be seen that the quality of emissivity image separated by RCE is poorer, and the image clarity is poor. However, the image clarity separated by $\alpha$ remaining method and NEM is better, the image texture is clear, and the emissivity information in detail reflects better. Whereas, the emissivity images retrieved by three methods contain some salt and pepper noise which interferes in the heat expression of the weak emissivity information.

Generally speaking, some statistical analysis methods are always used to evaluate the quality of image is good or bad, Evaluation methods include infor- 
mation quantity evaluation, clarity evaluation and fidelity evaluation [5].The paper adopted the method of information quantity evaluation to evaluate the quality of temperature and emissivity image separated by three methods.

The information quantity evaluation generally uses entropy and joint entropy to assess the image quality, entropy is an important indicator to assess the level of information richness, the greater the entropy is, the more abundant information is contained in the image, the image quality is better. U.S. Chavez (1984) proposed that the information entropy could be expressed by calculation formula of best index (OIF).The formula is as follows:

$$
\text { OIF }=\sum_{i=1}^{m} \sigma_{i} / \sum_{j=1}^{m}\left|R_{i j}\right|
$$

Wherein, $\sigma_{i}$ is the standard deviation of band $\mathrm{i}, R_{i j}$ is the correlation coefficient of band $i, j$. The standard deviation of image is greater , the information quantity is richer, the correlation coefficient in bands is smaller and the degree of information redundancy is smaller. Therefore, this formula chooses the data with large standard deviation and small correlation on the basis of statistical analysis, and hence, the OIF is larger, the information quantity of bands combination is greater .

The paper calculated the information entropy (OIF) of temperature and emissivity image separated by three methods mentioned above (Table 1.1).Judging from table 1.1,it is concluded that the information entropy of temperature image separated by RCM is largest, the amount of information in the image is richest, it is consistent with the subjective evaluation for the quality of temperature image separated by RCM. The information entropy of emissivity image separated by $\alpha$ remaining method is largest, the emissivity contrast effect of objects is best, however, its absolute precision of emissivity is poor. The information entropy of emissivity image separated by NEM is smaller than emissivity image separated by $\alpha$ remaining method, however, it is larger than emissivity image separated by RCM, furthermore, its absolute precision is highest. Therefore, which kind of emissivity image is chosen should combine with the actual demand. Above all, the temperature and emissivity image separated by three methods laid good foundation for the later objects recognition.

Table 1.1 Information entropy of temperature and emissivity image separated by three methods from TASI data.

\begin{tabular}{cc}
\hline Thermal infrared image & Information entropy \\
Temperature image separated by RCM & 484.386715 \\
Temperature image separated by NEM & 139.232229 \\
Emissivity image separated by RCM & $2.1253 \times 10^{-5}$ \\
Emissivity image separated by $\alpha$ remaining & 0.016137 \\
Emissivity image separated by NEM & $7.8 \times 10^{-4}$ \\
\hline
\end{tabular}




\subsection{Discussion}

From research results of paper, it can be clearly seen that the amount of information in the temperature image separated by RCM is largest, it is much better to identify the objects with week temperature difference. The absolute accuracy of temperature image separated by NEM is higher, It benefits for identifying the true temperature of objects. The contrast effect of emissivity image separated by $\alpha$ remaining method is best, it is much better to identify objects with week emissivity difference. The absolute accuracy of emissivity image separated by NEM is higher, It is good for identifying objects with low emissivity quantitatively. Therefore, which kind of temperature or emissivity image is chosen should combine with the actual demand. Above all, the paper established a set of temperature and emissivity separation and evaluation method which is suitable for anomaly heat identification of objects, what's more, the temperature and emissivity image separated by methods mentioned above laid good foundation for later objects recognition.

Acknowledgements Thank for the help of professor Jielin Zhang and Yingjun Zhao in the Beijing Research institute of Uranium Geology, National Key Laboratory of Remote Sensing Information and Image Analysis Technology.

\subsection{References}

1. Yang H., Zhang L F. (2011).Algorithm of emissivity spectrum and temperature separation based on TASI data. Journal of Remote Sensing, 15(6), pp.1248-1249.

2. Yang H., Zhang L F.(2010).Algorithm research of building materials emissivity extracting. IGARSS 2010, Honolulu, pp. 3350-3353.

3. Schmugge T., Hook S J \& Coll C.(1998).Recovering surface temperature and emissivity from thermal infrared multispectral data. Remote Sensing of Environment, 65(2),pp.121-131.

4. www.itres.com.Accessed 7 sept 2012.

5. Zhao Y S.(2002).The principles and methods of remote sensing application and analysis, Scientific Publishers, Bei jing in China,pp.110-115.

6. Johnson,B.R.(1998).IN-Scene atmospheric compensation: application to SEBASS data collected at the ARM site technical report. Space and Environment Technology Center, the Aerospace Corporation,pp.122-125.

7. Tian G L,Liu Q H.(2007).Thermal infrared remote sensing. Electronics Industry Publishers, Bei Jing in China, pp.172-174.

8. Kahle A.B.,Rowan L.C.(1980). Evaluation of multispectral middle infrared aircraft image from lithologic mapping in the east Tintic Mountains. Utah Geology,(8),pp.234-239.

9. Keakt P.S.,Gabell A.R.(1990). Estimation of emissivity and Temperature using Alpha coefficients. Proc. TIMS Workshop,JPL Publication, pp.90-95. 